\title{
Immune escape of AKT overexpressing ovarian cancer cells
}

\author{
JENS C. HAHNE ${ }^{1}$, SUSANNE R. MEYER ${ }^{1}$, STEPAN GAMBARYAN ${ }^{2}$, ULRICH WALTER $^{3}$, \\ JOHANNES DIETL ${ }^{1}$, JÖRG B. ENGEL ${ }^{4}$ and ARND HONIG ${ }^{1}$
${ }^{1}$ Department of Gynecology, University Hospital of Würzburg; ${ }^{2}$ Institute for Clinical Biochemistry and Pathobiochemistry, University of Wuerzburg, D-97080 Würzburg; ${ }^{3}$ Centre for Thrombosis and Haemostasis, University of Mainz, D-55131 Mainz; ${ }^{4}$ Department of Gynecology, University of Regensburg, D-93055 Regensburg, Germany

Received December 6, 2012; Accepted February 4, 2013

DOI: 10.3892/ijo.2013.1846

\begin{abstract}
Platinum-resistance is the most crucial problem for treatment of ovarian cancer. There is a clinical need for new treatment strategies which overcome platinum resistance. As survival is strongly influenced by immunological parameters, immunotherapeutic strategies appear promising. Therefore a better understanding of the interaction between ovarian tumour cells and cells of the immune system is a necessary prerequisite. In the present study we aimed to enlighten the interactions between platinum resistant and platinum sensitive ovarian cancer cells and natural-killer (NK)-cells. Modified FATAL assay was used for determining the killing efficiency of NK-cells for the parental A2780 cells and the cis-platinum resistant A2780cis human ovarian cancer cells. Expression of pro- and anti-apoptotic genes as well as ligands involved in NK-cell receptor recognition were analysed by RT-PCR and flow cytometric analysis. The efficiency of NK mediated cell lysis differs between A2780 cells and the cis-platinum-resistant A2780cis cells. A2780cis cells are less accessible for NK-cell mediated killing. Based on this observation we characterized the molecular basis for resistance mechanisms. Besides an increase in anti-apoptotic genes (especially CIAP-1 and -2) that probably render A2780cis cells more resistant against apoptosis an increased amount of soluble MICA/B seems to be responsible for the lower killing rate of platinum-resistant A2780cis cells compared to their parental A2780 cells.
\end{abstract}

\section{Introduction}

Ovarian cancer is the most common cause of cancer death from gynecologic tumours and currently causes $\sim 100,000$ deaths per year world-wide (1). While treatment of ovarian cancer with platinum-based agents has been established for decades, these still remain the most active substances for this

Correspondence to: Dr Arnd Honig, Department of Gynecology, University Hospital of Würzburg, Josef-Schneider-Str. 4, D-97080 Würzburg, Germany

E-mail: arnd_hoenig@hotmail.com

Key words: ovarian cancer, AKT, platinum-resistance, natural-killer cells entity (2). Accordingly, primary resistance to platinum-based therapy is associated with a worse disease-free and overall survival (3). However, virtually all patients eventually develop secondary resistance to platinum based agents and compounds used for second- or third-line treatment. Other substances display substantially less anticancer activity as compared to platinum (4). There is a definite clinical need to develop new treatment strategies to overcome platinum resistance. As survival is strongly influenced by immunological parameters, immunotherapeutic strategies appear promising, therefore a better understanding of the interaction between ovarian tumour cells and cells of the immune system is necessary.

Natural-killer (NK)-cells play an important role in immune surveillance and co-ordinating responses of other immune cells. Most tumour cells express surface molecules that can be recognized by activating receptors on NK-cells (5). The expression of these receptors make such cells susceptible to endogenous NK-cells, but malignant cells have developed mechanisms to evade innate immune surveillance (6-8). In patients with cancer, it is presumed that tumour cells have developed mechanisms to suppress NK-cell activation and resist lysis by endogenous NK-cells, but the molecular basis for tumour cell resistance against this lysis is not well understood.

Alterations of the serine/threonine kinase AKT/PKB pathway have been detected in several human malignancies including ovarian cancer (9). AKT has a broad range of downstream effectors that regulate cell processes such as cell growth, cell cycle progression, survival, migration, and angiogenesis (10). The AKT pathway is a promising target for cancer therapy, as it is a main nodal point where extracellular and intracellular oncogenic signals are integrated. Due to the key role of AKT in malignant transformation numerous inhibitors of the AKT-pathway have been developed, and are currently in various stages of clinical development (11).

In human specimens of ovarian cancer AKT was found to be activated in $68 \%$ (9) and PI3K, an upstream component of the AKT-pathway, was found to be mutated in $12 \%$ of the cases (12). Recent evidence by our group and others has shown that overactivation of the AKT-pathway may be associated with platinum resistance (13-17). It was demonstrated that parental A2780 cells become platinum resistant by overexpression of AKT and that platinum resistance in A2780cis cells can be overcome by transfection with siRNA downregulating AKT (17). 
In the present study we enlightened the interaction between ovarian tumour cells with different AKT expression levels and NK-cells.

\section{Materials and methods}

Cell culture. A2780 and A2780cis cell lines (both are p53 and KRAS wild-type cell lines) were obtained from ECACC (Salisbury, UK). The cis-platinum-resistant A2780cis cell line has been developed by chronic exposure of the parental cis-platinum-sensitive A2780 cell line to increasing concentrations of cis-platinum (18).

Preparation of cell lysates and western blotting. Preparation of cell lysates was performed as previously described (17). Membranes were probed overnight with anti-phospho-Akt antibody from Epitomics (Burlingame, CA, USA), antiB7-H1 antibody from eBioscience (Frankfurt, Germany) or anti- $\beta$-actin antibody from Abcam (Cambridge, UK), respectively. Secondary horseradish peroxidase (HRP)-conjugated antibodies were from Cell Signaling (Frankfurt, Germany). The chemiluminescent HRP substrate solution (Millipore, Schwalbach, Germany) was used for detection.

NK-cell preparation and lysis assay. PBMC were obtained from healthy volunteers by density gradient centrifugation (Biocoll; Biochrom AG, Berlin, Germany). Monocytes were depleted by adherence and the remaining non-adherent PBL were further cultured on irradiated (30 Gy) RPMI-8866 feeder cells to obtain polyclonal NK-cell populations (19). After 6 days of co-culture 500 U IL-2 (Peprotech, Hamburg, Germany) were added per $\mathrm{ml}$ and $48 \mathrm{~h}$ later the polyclonal NK-cell population (effector cells) was used in different killing assays. Therefore, the NK-cells were labeled with eFluor 670 (eBioscience) and lytic activity against CFSE-stained (eBioscience) tumour cells (targets; $10^{5}$ cells/well) was assessed in a modified $5 \mathrm{~h}$ FATAL assay using various effector:target ratios (20). Cells were detached by trypsinisation and target cell lysis was determined by flow cytometric analysis of 30,000 target cells in a FACScan flow cytometer (Calibur, BD Biosciences, Heidelberg, Germany). eFluor 670-negative target cells were selected by gating and the percentage of CFSE cells within this population was determined. Spontaneous leakage of CFSE was determined by incubating the target cells with medium alone.

Flow cytometry analysis. Cells/sample $\left(10^{6}\right)$ were detached with Accutase (PAA, Cölbe, Germany), blocked and stained with Alexa Fluor ${ }^{\circledR} 488$ conjugated anti-human MICA/B antibody (eBioscience) or with the relevant isotype control according to the manufacturer's instructions.

$R T-P C R$. Total cellular RNA was extracted from cells by RNeasy kit (Qiagen, Hilden, Germany). The quality and quantity of RNA preparations was assessed with NanoDrop ND-1000 (PEQLAB Biotechnologie GmBH, Erlangen, Germany). Generation of cDNAs by reverse transcription and RT-PCR reaction was performed in one step (Qiagen OneStep RT-PCR kit; Qiagen) according to the manufacturer's instructions. The gene for the constitutively expressed ribo- somal protein L13A (rpL13A) was used as housekeeping gene (21) in order to monitor RNA quality and cDNA synthesis, and to ensure that equivalent amounts of cDNA were used in all PCR amplifications. Oligonucleotides were synthesized by Sigma-Aldrich (Taufkirchen, Germany): rpL13A f, 5'-TACGC TGTGAAGGCATCAAC-3'; rpL13Ar, 5'-CACCATCCGCTTT TTCTTGT-3'; $b c l$-2 f, 5'-ATGGCG CACGCTGGGAGAAC-3'; $b c l-2$ r, 5'-GCATGCTGGGGCCGTACAGT-3'; $b c l-x L$ f, 5'-CGG TGAATGGAGCCACTGCG-3'; $b c l-x L$ r, 5'-GTCACTGAATG CCCGCCGGT-3'; $b c l-w$ f, 5'-CCCAGGCTCAGCCCAGC AAC-3'; $b c l-w$ r, 5'-CCCAGTTCCCCTCCCGCAGA-3'; ciap-1 f, 5'-AGCCTGCTTTGCCTGTGGTGG-3'; ciap-1 r, 5'-GCCG CAGCATTTCCTTTAACCCA-3'; ciap-2 f, 5'-GCCTTGATG AGAAGTTCCTACCCCT-3'; ciap-2 r,5'-AGCCCATTTCCAC GGCAGCA-3'; bim f, 5'-CAGCCACCCTGCGAACCCTG-3'; bim r, 5'-GGGCAGCTGTCCCCTTCACC-3'; bak f, 5'-ACGG CAGCTCGCCATCATCG-3'; bak r, 5'-GAAGAGCCACC ACACGGCCC-3'; bax f, 5'-TGATGGACGGGTCCGG GGAG-3'; bax r, 5'-GGGGAGAGGGCACCACTGTGA-3'; PVRL2 (coding for CD112)1; f, 5'-GGACCCTGGCCGGAACT GTC-3'; PVRL2 r, 5'-AATGATGGCGGCGATGATGCCC-3'; $P V R$ (coding for CD155) f, 5'-CTGATCCTGCTGGGGAT CGGG-3'; PVR r, 5'-CCCCTCTCAGTCCCGACGCT-3'; ulbpl f, 5'-CCCCGCGTTCCTTCTGTGCC-3'; ulbpl r, 5'-TAG ACAGGCGGCCTCCCTGAA-3'; $u l b p 2$ f, 5'-CCACGGTGGT GTGCGGTTCA-3'; ulbp2 r, 5'-TGGCCAGACAGAAGGG CGAGT-3'; ulbp3 f, 5'-CTTCCGCGCCTCGCGATTCT-3'; ulbp3 r, 5'-AGTCTGAGCCTCTGCCCCACC-3'; MICA f, 5'-CTGCCTGCAGGAACTACGGCG-3'; MICA r, 5'-AGCAG CCAGCAGCAACAGCAGAA-3'; $M I C B$ f, 5'-GAGCGGGGC GCAGGTGACTAA-3'; $M I C B$ r, 5'-AGCGCAGGAAGGGCT GACCA-3'; mmp-9 f, 5'-TTGACAGCGACAAGAAGTGG-3'; mmp-9 r, 5'-GCCATTCACGTCGTCCTTAT-3'; adam10 f, 5'-GGCGGGGATGGGAGGTCAGT-3'; adam10 r, 5'-AGGT GCTCCAACCCAAGCCA-3'; adam17f,5'-GCTGCAACAGC GACTGCACG-3'; adam17 r, 5'-GCGCCGAAGGGATCAC AGGG-3'; timp-3 f, 5'-AAAGGAGGGGCCCTTCGGCA-3'; timp-3 r, 5'-CTTCTGCCGGATGCAGGCGT-3'. All PCR products were analyzed by separation on a $2 \%$ agarose gel stained with GelRed (Biotium, Hayward, CA, USA).

\section{Results}

This study focused on natural-killer (NK)-cells and their interaction with a platinum-sensitive parental human ovarian cancer cell line A2780 and the corresponding platinum-resistant cell line A2780cis. These two cell lines were used because they are well characterized and furthermore they have the same genetic background but differ with regard to platinum resistance. First of all the different pAKT expression levels were analyzed in the two cell lines by western blotting (Fig. 1). The expression of pAKT is strongly induced in A2780cis cells in comparison to the expression in the parental A2780 cells.

Next the killing efficiency of NK-cells, prepared as described in the Material and methods, were confirmed with a luciferase-based assay in the reporter cell line K562 (data not shown). After their killing capacity was established, the NK-cells were used in the modified FATAL assay with A2780 and A2780cis cells. As shown in Fig. 2 the killing rates of tumour cells differ significantly between the two cell lines. 


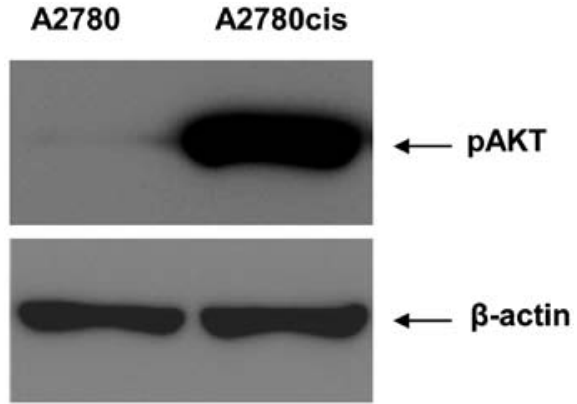

Figure 1. Western blotting showing expression of phosphorylated AKT-1 (pAKT) protein. In each lane $20 \mu \mathrm{g}$ of total protein was analysed by $10 \%$ SDS-polyacrylamide gel electrophoresis and transferred onto nitrocellulose. Membranes were probed overnight with anti-phospho-Akt antibody (Epitomics), then with a horseradish peroxidase-conjugated secondary antibody (Cell Signaling). Antibodies were detected using chemiluminescent HRP substrate solution (Millipore). The housekeeping protein $\beta$-actin was used as internal control.

Parental A2780 cells seem to be better targets for NK-cell mediated killing than the A2780cis cells at all ratios between NK-cells and tumour cells used in this experiment. Only if 10 times more NK-cells than tumour cells are used the killing rate of A2780cis cells reached nearly that of wild-type A2780 cells. Similar results were obtained with NK-cell preparations derived from other healthy donors. Therefore the observed differences in the killing rate between A2780 and A2780cis cells are neither dependent on the healthy donor nor the NK-cell preparation.

Next we addressed the question why the interaction and killing between NK-cells and A2780 cells is much better. B7-H1 (programmed cell death 1 ligand 1) ligand for the receptor PD1 (programmed death 1) protects cells against NK-cell mediated lysis, because the interaction of B7-H1 with PD1 results in inhibition of T-cell-receptor-mediated proliferation and cytokine production. However, as shown in Fig. 3 neither A2780 (Fig. 3, lane 1) nor A2780cis cells (Fig. 3, lane 2) express the B7-H1 ligand. Likewise B7-H1 was not expressed in the ovarian cancer cell line OAW42 (Fig. 3, lane 3). In contrast the B7-H1 protein can be detected with the specific antibody in other ovarian cancer cell lines, e.g., SKOV-3 (Fig. 3, lane 4) and OVCAR-3 (Fig. 3, lane 5), which were used as positive controls in this experiment. Therefore, B7-H1 ligand seems

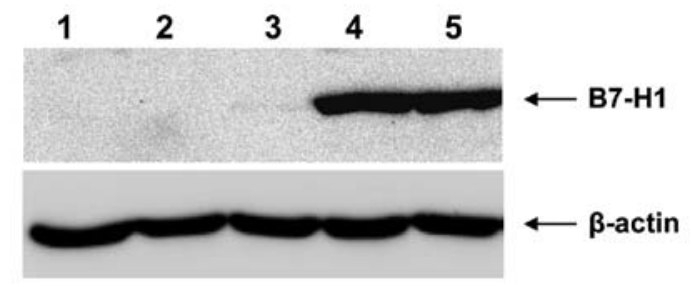

Figure 3. Western blotting showing expression of B7-H1 protein. In each lane $20 \mu \mathrm{g}$ of total protein (lane 1,A2780; lane 2, A2780cis; lane 3, OAW-42; lane 4, SKOV-3; lane 5, OVCAR-3) was analysed by $10 \%$ SDS-polyacrylamide gel electrophoresis and transferred onto nitrocellulose. Membranes were probed overnight with anti-B7-H1 antibody (eBioscience), then with a horseradish peroxidase-conjugated secondary antibody (Cell Signaling). Antibodies were detected using chemiluminescent HRP substrate solution (Millipore). The housekeeping protein $\beta$-actin was used as internal control.

not to be involved in the observed different NK-cell mediated lysis rates.

In the next step the expression rates of different anti- and pro-apoptotic genes were studied by RT-PCR (Fig. 4). The expression rate of the anti-apoptotic gene $b c l-2$ is decreased in A2780 cis cells compared to parental A2780 cells. The antiapoptotic genes $b c l-x L$ and $b c l-w$ were expressed nearly at the same rate in the two cell lines. Obviously the expression of the anti-apoptotic genes cellular inhibitor of apoptosis (ciap) -1 and -2 is strongly induced in A2780cis cells compared to A2780 cells. The pro-apoptotic genes such as bim and bak showed no expression differences in the two cell lines but bax expression is decreased in the platinum-resistant cell line. Also the expression rate of CD112 and CD155, both ligands for the NK-cell activating receptor DNAM-1, were analyzed. There was no difference in CD155 expression in the two cell lines and CD112 expression was slightly increased in A2780 cells. The expression rate for NKG2D ligands, e.g., MICB, ULBP1, ULBP2 and ULBP3, were increased in A2780cis cells compared to the parental A2780 cells. Even if the expression rate of MICA was found to be nearly the same in both cell lines we decided to analyse the expression differences of MICA and MICB between A2780 and A2780cis cells, because these two proteins are highly related and both activate cytotoxicity by NK-cells through the NKG2D receptor as a mechanism of immunological defence. Furthermore, shedding of MICA and MICB seems to be one of the mechanisms by which tumours

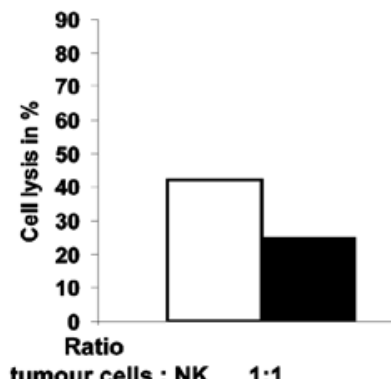

tumour cells : NK 1:1

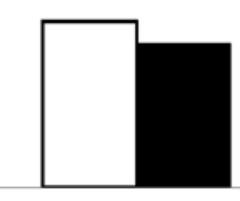

$1: 2$

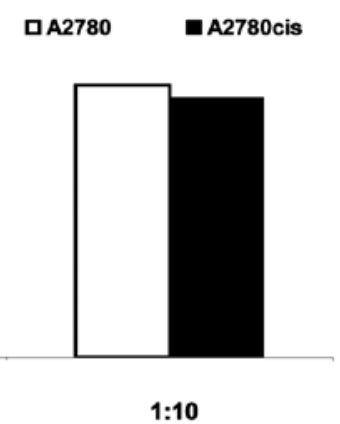

$1: 5$

$1: 10$

Figure 2. Lytic activity of polyclonal NK-cells. A2780 and A2780cis cells ( $10^{5}$ cells/well), respectively, were used as targets in a modified 5 h FATAL assay using various tumour cell:NK-cell ratios. Target cell lysis was determined by flow cytometric analysis. The percentage of tumour cell lysis was determined in relation to a control containing tumour cells with medium alone. A representative of three independent experiments is shown. 


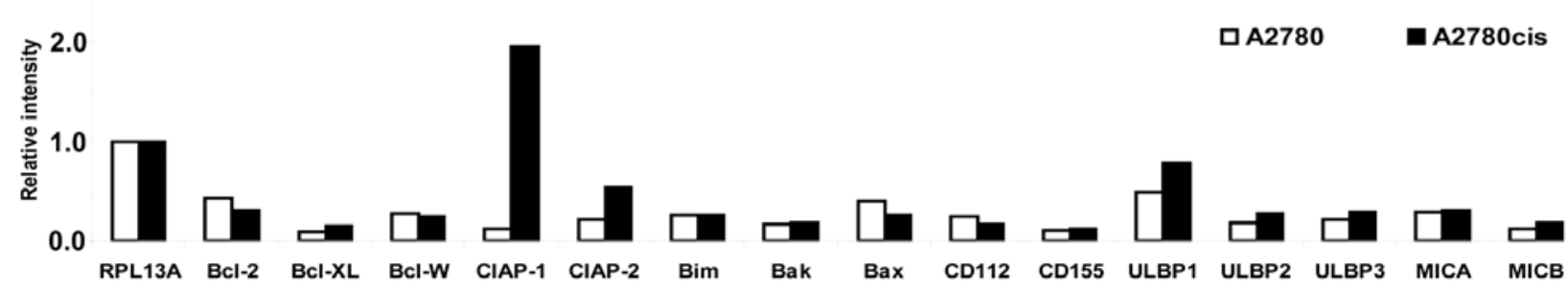

Figure 4. Quantification of the PCR products by densitometric measurement. The expression rate of the housekeeping gene RPL13A was equal in A2780 and A2780cis cells and was set as one. The expression rates of different anti- and pro-apoptotic genes as well as some genes coding for known ligands of the NK-cell receptors DNAM-1 and NKG2D were related to the control.
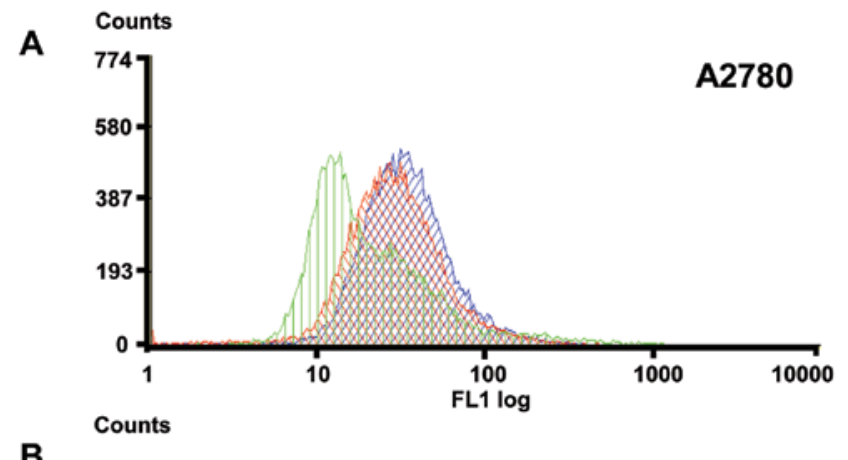

B

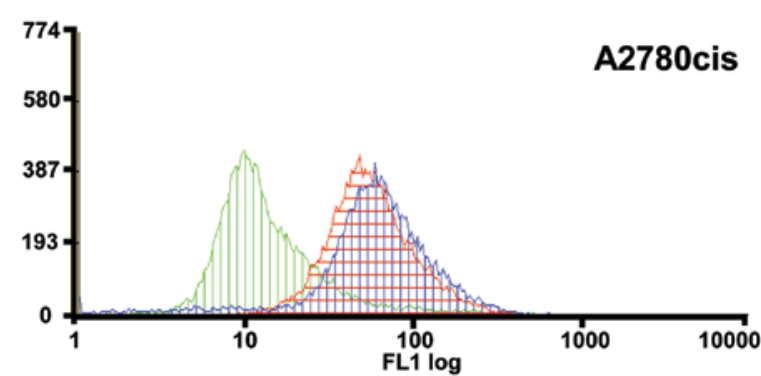

Figure 5. Surface expression of MICA/B on A2780 and A2780cis cells. (A) A2780 and (B) A2780cis cells were stained with anti-MICA/B antibody after incubation without (red graph) or with (blue graph) addition of the protease inhibitor GM6001 for $24 \mathrm{~h}$. Addition of protease inhibitor GM6001 results in a 5.3\% increased presence of MICA/B protein on the A2780cis cell surface and $<0.1 \%$ increase on the cell surface of A2780 cells as shown in a logarithmic scale. The respective isotype control (green graph) was not influenced by preincubation with GM6001.

evade host immune surveillance. Expression of MICA/B was analyzed by FACS with and without addition of GM6001 a broad-spectrum inhibitor of proteinases (Fig. 5). Pre-incubation of A2780cis cells with GM6001 results in 5.3\% higher amount of MICA/B protein on the cell surface compared to untreated A2780cis cells. Therefore it is very likely that A2780cis cells could be protected against NK-cell mediated lysis by shed soluble MICA/B. In contrast the amount of MICA/B protein on the surface of parental A2780 cells is not significantly influenced (increased $<0.1 \%$ ) by pre-incubation with the proteinase inhibitor. This observation led us to have a look on the expression pattern of different proteinases especially MMP-9, ADAM-10 and ARAM-17 known to be involved in cleavage of MICA/B as well as TIMP3 a known inhibitor of the proteolysis shedding process (Fig. 6). As illustrated in Fig. 6 the expression rate of MMP-9 is slightly decreased in A2780cis cells compared to the parental A2780 cells whereas the expression of ADAM-10 and ADAM-17 is the same in both cell lines. Expression of TIMP3 is significantly reduced in A2780cis cells compared to A2780 cells and therefore the proteolytic shedding of MICA/B is probably more pronounced in the A2780cis cell line.

\section{Discussion}

Natural-killer (NK)-cells are a critical component of the innate immune response against infectious pathogens and malignant transformation $(22,23)$. NK-cells mediate this activity through the elaboration of various cytokines as well as through direct cytolytic activity. However, unlike adaptive immune cells, which utilize specific clonal recognition receptors, NK-cell activation depends on a complex balance between activating and inhibitory signals $(24,25)$. Nevertheless, NK-cells play an important role in immune surveillance and coordinating responses of other immune cells. Most tumour cells express surface molecules that can be recognized by activating receptors on NK-cells (5). The expression of these receptors

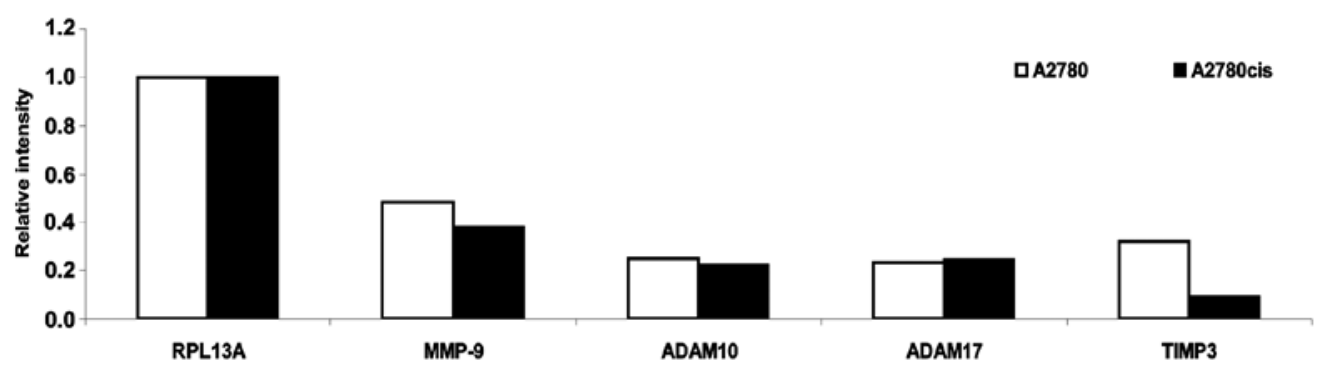

Figure 6. Quantification of the PCR products by densitometric measurement. The expression rate of the housekeeping gen RPL13A was equal in A2780 and A2780cis cells and was set as one. The expression rates of genes coding for proteinases known to be involved in cleavage of MICA/B as well as TIMP3 a known inhibitor of the proteolysis shedding process were related to the control. 
make such cells susceptible to endogenous NK-cells, but malignant cells have developed mechanisms to evade these mechanisms of innate immune surveillance (6-8). In patients with cancer, it is presumed that tumour cells have developed mechanisms to suppress NK-cell activation and resist lysis by endogenous NK-cells, but the molecular basis for target resistance is not well understood. The goal of our studies was to characterize the molecular basis for these resistance mechanisms in ovarian cancer with different AKT expression levels. Therefore the interactions of NK-cells with a platinum-sensitive parental human ovarian cancer cell line and the corresponding platinum-resistant cell line was analyzed. These cell lines have the same genetic background but differ with regard to the AKT expression level. We found great differences in the efficiency of NK mediated cell lysis between the cell lines. The cis-platinum-resistant A2780cis cells are less accessible for NK-cell mediated killing. This finding is in agreement with a recent report by Bellucci et al (26). Using a lentiviral shRNA library targeting $>1,000$ human genes they identified 83 genes that promote target cell resistance to human NK-cell-mediated killing (26). Many of the genes identified in this genetic screen belong to common signalling pathways including members of the AKT/PI3Kpathway such as PIK3CA and PIK3CB (26).

The comparison of cancer cell lines A2780 and A2780cis revealed that the observed differences with regard to NK-cell mediated killing are most probably based on two mechanisms. First of all the observed increased expression of anti-apoptotic genes (especially ciap-1 and -2) in A2780cis cells compared to A2780 cells most probably renders A2780cis cells more resistant against apoptosis. Second the CD112 ligand for NK-cell receptor DNAM-1 was expressed at a reduced level in A2780cis cells but ligands for the NK-cell receptor NKG2D were expressed more strongly in the platinum-resistant cells compared to parental A2780 cells.

A2780cis cells express lower levels of TIMP-3 the inhibitor of MICA/B shedding. At the same time the proteases for shedding are expressed which result in a net increase of soluble MICA/B in A2780cis cell cultures as shown by FACS analysis in this study. It is well known that cleaved MICA/B protect cells against NK mediated cell killing $(27,28)$. Therefore, we conclude that most probably the increased amount of soluble MICA/B is responsible for the lower killing rate of platinum-resistant A2780cis cells compared to their parental A2780 cells. Previously it was demonstrated that PI3K/AKT pathway is involved in inducing MICA/B expression in breast cancer cells (29). Our results revealed that the cells with an increase in phosphorylated AKT/activated PI3K/AKT pathway have a higher MICA/B expression. Recently Bellucci et al demonstrated that treatment of tumour cells with JAK inhibitors increased their susceptibility to NK-cell mediated killing (26). The authors concluded that common signalling pathways can regulate susceptibility of human tumour cells to killing by immunologic effector cells and that small molecule inhibitors of these kinases may have important immunologic effects in vivo (26). Whether in analogy inhibition of PI3K/ AKT pathway renders the platinum-resistant A2780cis cells accessible for NK-cell mediated killing must be evaluated in further studies. Our study presented here characterizes the molecular basis for resistance mechanisms in ovarian cancer with different AKT expression levels regarding NK-cell mediated killing. In conclusion, the cis-platinum-resistant A2780cis cells are less accessible for NK-cell mediated killing in comparison to parental A2780 cells. Different mechanisms seem to be relevant; first an increased expression of anti-apoptotic genes (especially ciap- 1 and -2 ) and second an increase of soluble MICA/B seems to be present in cisplatinum-resistant cells.

\section{Acknowledgements}

We appreciate the permission to use the INTAS ChemoStar Imager (Department of Microbiology, University of Würzburg), and we thank especially Professor T. Rudel and Dr B. Bergmann. This study was supported by IZKF Würzburg.

\section{References}

1. Jemal A, Siegel R, Xu J and Ward E: Cancer statistics, 2010. CA Cancer J Clin 60: 277-300, 2010.

2. Pignata S, Cannella L, Leopardo D, Pisano C, Bruni GS and Facchini G: Chemotherapy in epithelial ovarian cancer. Cancer Lett 303: 73-83, 2011.

3. Gonzalez-Martin AJ: Medical treatment of epithelial ovarian cancer. Expert Rev Anticancer Ther 4: 1125-1143, 2004.

4. Matsuo K, Lin YG, Roman LD and Sood AK: Overcoming platinum resistance in ovarian carcinoma. Expert Opin Investig Drugs 19: 1339-1354, 2010.

5. Lanier LL: NK cell recognition. Annu Rev Immunol 23: 225-274, 2005.

6. Dunn GP, Bruce AT, Ikeda H, Old LJ and Schreiber RD: Cancer immunoediting: from immunosurveillance to tumor escape. Nat Immunol 3: 991-998, 2002.

7. Orr MT and Lanier LL: Natural killer cell education and tolerance. Cell 142: 847-856, 2010.

8. Smyth MJ, Dunn GP and Schreiber RD: Cancer immunosurveillance and immunoediting: the roles of immunity in suppressing tumor development and shaping tumor immunogenicity. Adv Immunol 90: 1-50, 2006.

9. Altomare DA, Wang HQ, Skele KL, et al: AKT and mTOR phosphorylation is frequently detected in ovarian cancer and can be targeted to disrupt ovarian tumor cell growth. Oncogene 23: 5853-5857, 2004

10. Cheng JQ, Lindsley CW, Cheng GZ, Yang H and Nicosia SV: The Akt/PKB pathway: molecular target for cancer drug discovery. Oncogene 24: 7482-7492, 2005.

11. Steelman LS, Chappell WH, Abrams SL, et al: Roles of the Raf/ MEK/ERK and PI3K/PTEN/Akt/mTOR pathways in controlling growth and sensitivity to therapy- implications for cancer and aging. Aging 3: 192-222, 2011.

12. Levine DA, Bogomolniy F, Yee CJ, et al: Frequent mutation of the PIK3CA gene in ovarian and breast cancers. Clin Cancer Res 11: 2875-2878, 2005.

13. Benedetti V, Perego P, Luca Beretta G, et al: Modulation of survival pathways in ovarian carcinoma cell lines resistant to platinum compounds. Mol Cancer Ther 7: 679-687, 2008.

14. Engel JB, Schonhals T, Hausler S, et al: Induction of programmed cell death by inhibition of AKT with the alkylphosphocholine perifosine in in vitro models of platinum sensitive and resistant ovarian cancers. Arch Gynecol Obstet 283: 603-610, 2011.

15. Santiskulvong C, Konecny GE, Fekete M, et al: Dual targeting of phosphoinositide 3-kinase and mammalian target of rapamycin using NVP-BEZ235 as a novel therapeutic approach in human ovarian carcinoma. Clin Cancer Res 17: 2373-2384, 2011.

16. Westfall SD and Skinner MK: Inhibition of phosphatidylinositol 3-kinase sensitizes ovarian cancer cells to carboplatin and allows adjunct chemotherapy treatment. Mol Cancer Ther 4: 1764-1771, 2005.

17. Hahne JC, Honig A, Meyer SR, et al: Downregulation of AKT reverses platinum resistance of human ovarian cancers in vitro. Oncol Rep 28: 2023-2028, 2012. 
18. Behrens BC, Hamilton TC, Masuda H, et al: Characterization of a cis-diamminedichloroplatinum(II)-resistant human ovarian cancer cell line and its use in evaluation of platinum analogues. Cancer Res 47: 414-418, 1987.

19. Valiante NM, Rengaraju M and Trinchieri G: Role of the production of natural killer cell stimulatory factor (NKSF/IL-12) in the ability of B cell lines to stimulate T and NK cell proliferation. Cell Immunol 145: 187-198, 1992.

20. Sheehy ME, McDermott AB, Furlan SN, Klenerman P and Nixon DF: A novel technique for the fluorometric assessment of T lymphocytic antigen specific lysis. J Immunol Methods 249: 99-110, 2001

21. Jesnowski R, Backhaus C, Ringel J and Lohr M: Ribosomal highly basic $23-\mathrm{kDa}$ protein as a reliable standard for gene expression analysis. Pancreatology 2: 421-424, 2002.

22. Caligiuri MA: Human natural killer cells. Blood 112: 461-469, 2008.

23. Vivier E, Raulet DH, Moretta A, et al: Innate or adaptive immunity? The example of natural killer cells. Science 331: 44-49, 2011.
24. Lanier LL: Up on the tightrope: natural killer cell activation and inhibition. Nat Immunol 9: 495-502, 2008.

25. Moretta L, Biassoni R, Bottino C, Mingari MC and Moretta A: Human NK-cell receptors. Immunol Today 21: 420-422, 2000.

26. Bellucci R, Nguyen HN, Martin A, et al: Tyrosine kinase pathways modulate tumor susceptibility to natural killer cells. J Clin Invest 122: 2369-2383, 2012.

27. Boutet $\mathrm{P}$, Agüera-Gonza'lez S, Atkinson S, et al: Cutting edge: the metalloproteinase ADAM17/TNF- $\alpha$-converting enzyme regulates proteolytic shedding of the MHC class I-related chain B protein. J Immunol 182: 49-53, 2009.

28. Waldhauer I, Goehlsdorf D, Gieseke F, et al: Tumor-associated MICA is shed by ADAM proteases. Cancer Res 68: 6368-6376, 2008.

29. Okita R, Mougiakakos D, Ando T, et al: HER2/HER3 signaling regulates NK cell-mediated cytotoxicity via MHC class I chainrelated molecule $\mathrm{A}$ and $\mathrm{B}$ expression in human breast cancer cell lines. J Immunol 188: 2136-2145, 2012. 